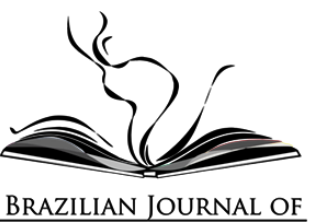

\title{
COOPERACIÓN E INTEGRACIÓN ECONÓMICA INTERNACIONAL ENTRE CHINA Y COLOMBIA
}

\author{
COOPERATION AND INTERNATIONAL ECONOMIC INTEGRATION \\ BETWEEN CHINA AND COLOMBIA
}

COOPERAÇÃO E INTEGRAÇÃO ECONÔMICA INTERNACIONAL ENTRE CHINA E COLÔMBIA

Bernardo Romero-Torres ${ }^{7}$ (i) Andrés Escobar-Espinoza ${ }^{2}$ iD Universidad de Cartagena, Colombia

Resumen: El documento presenta una discusión de la evolución de la integración económica entre Colombia y China en el siglo XXI desde distintas perspectivas: evolución del comercio exterior con énfasis en las principales exportaciones e importaciones; comportamiento de la inversión extranjera directa (IED), teniendo en consideración los acuerdos de cooperación técnica, económica y cultural bajo el esquema de cooperación internacional Sur-Sur; evidencia de un interés conjunto en avanzar hacia una integración económica profunda que favorezca la libre circulación de bienes y servicios. Además, se analizan los beneficios potenciales de una mayor integración económica entre ambos países, y se confirma que la economía China se considera como un actor relevante en el desarrollo económico de Colombia. Por último, se destacan los principales retos que debe enfrentar Colombia en este escenario de mayor integración y cooperación internacional.

\footnotetext{
I Investigador del Grupo de Investigación Economía Aplicada de la Universidad de Cartagena. Mail: bromerot@unicartagena.edu.co.

${ }^{2}$ PhD en Economía de la Universita Cattolica del Sacro Cuore (Italia). Actualmente, Coordinador Académico General e Investigador del Grupo de Investigación Economía Aplicada de la Universidad de Cartagena. Mail: aescobare@unicartagena.edu.co.
} 
Palabras clave: Relaciones internacionales; Integración Económica; Acuerdos de Cooperación.

Abstract: The paper presents an assessment of the recent evolution of the economic integration between Colombia and China during the 21th century from different perspectives: evolution of international trade with emphasis on exports and import, the behavior of foreign direct investment (FDI) considering technical, economic, and cultural cooperation agreements under the scheme of South-South cooperation, towards a more profound international economic integration that favors the free movement of goods and services. In addition, the potential benefits of this economic integration are analyzed and conclude that China is an important actor in the economic development prospects of Colombia. Finally, the paper emphasizes the main challenges ahead in a scenario of international economic integration.

Keywords: International Relations; Economic Integration; Cooperation Agreements

Resumo: Este artigo apresenta uma discussão sobre a evolução da integração econômica entre Colômbia e China no século XXI sob diferentes perspectivas: evolução do comércio exterior com ênfase nas principais exportações e importações; comportamento do investimento estrangeiro direto (IED) levando em consideração acordos de cooperação técnica, econômica e cultural no âmbito do esquema de cooperação internacional Sul-Sul/ e que demonstrem interesse conjunto em avançar para uma integração econômica profunda que favoreça a livre circulação de bens e serviços. Além disso, são analisados os benefícios potenciais de uma maior integração econômica entre os dois países, e se confirma que a economia chinesa é considerada um ator relevante no desenvolvimento econômico da Colômbia. Por fim, destacam-se os principais desafios que a Colômbia 
deve enfrentar neste cenário de maior integração e cooperação internacional.

Palavras-chave: Relações Internacionais; Integração Econômica; Acordos de Cooperação.

\section{INTRODUCCIÓN}

A pesar de la Crisis Financiera Global (CFG) de 2008, el crecimiento económico de China ha sido sorprendente $y$, junto a otros países emergentes, ha liderado la recuperación de la economía mundial. Los resultados económicos de China de la última década contrastan con los obtenidos por los países más avanzados. Aunque su crecimiento económico se ha desacelerado desde el año 2010, pasando de 10,6\% a 6,6\% para el año 2018 según los datos del Banco Mundial, sigue siendo un pilar fundamental para el desarrollo de la economía mundial.

China cuenta con una infraestructura que permite la interconexión entre grandes complejos industriales que producen maquinaria y equipos electrónicos para ser vendidos a consumidores alrededor del mundo, al mismo tiempo que compran insumos y materias primas al resto que sirven para la producción del sector manufacturero. Además, como una población superior a la colombiana logró superar la pobreza, cuenta con una nueva clase media de más de trecientos millones de personas que demandan distintos bienes de consumo, incluso a través de medios tecnológicos como lo es el comercio electrónico. De esta manera, ha logrado una mayor oferta de sus productos transables y un aumento en la demanda y en los precios internacionales de materias primas. 
El comercio de China se ha consolidado como uno de los más importantes a nivel mundial. El flujo comercial de China con el resto del mundo ha aumentado inclusive superando a los Estados Unidos, Japón y Alemania, y se sitúa como la economía que más comercia a nivel mundial. Este creciente rol de China en el comercio exterior, ha sido impulsado por su transformación productiva, una mayor integración a los mercados y la atracción de Inversión Extranjera Directa (ROMERO; LÓPEZ; ESCOBAR, 2017).

El objetivo principal de este documento es analizar la evolución de la integración económica entre Colombia y China teniendo en cuenta la importancia que tiene en la dinámica comercial entre las dos economías. En la siguiente sección se discute acerca de la hegemonía y el comercio internacional. Posteriormente, se presenta el rol de China como potencia emergente en el crecimiento global y en particular del colombiano, luego se presentan las principales relaciones económicas entre China y Colombia, y los beneficios de una mayor integración económica. En la última sección finalmente se presentan las conclusiones y recomendaciones.

\section{HEGEMONÍA, LIDERAZGO Y COMERCIO INTERNACIONAL}

La hegemonía es el dominio de un estado en particular, en donde éste tiene el poder de hacer lo que desea y los más débiles deben obedecer (JOSEPH, 2017). Cuando un Estado despliega su poder hegemónico ejerciendo el liderazgo por medio de la diplomacia, persuasión o coerción puede generar estabilidad en el sistema internacional porque tiene la capacidad de establecer ciertas reglas internacionales para un ordenado funcionamiento del sistema y puede sancionar a los posibles infractores (YAZID, 2015). En esto consiste la Teoría de la Estabilidad Hegemónica (TEH) que tiene como tesis que la distribución del poder entre los estados es el 
determinante principal del sistema económico internacional y es más propicio a ser estable (WEBB; KRASNER, 1989).

En cuanto a las relaciones económicas internacionales, es difícil diferenciar el dominio del liderazgo. El término líder puede ser agrupado bajo el concepto de líder pasivo y líder activo, que están normalmente relacionados con los tipos de liderazgos que son reservados para un estado hegemónico (WIENER, 1995). El liderazgo pasivo hace referencia a que un estado es más avanzado que otros estados (ya sea en términos tecnológicos, científicos, entre otros), y que puede liderar en términos de producción, comercio e inversión tanto con ventajas competitivas como dinamismo de la actividad comercial, proporcionando así estímulos económicos. En cambio, el liderazgo activo, hace referencia al acto de proveer la estructura normativa y autoritativa del sistema internacional.

En ese sentido, y teniendo en cuenta el término liderazgo pasivo, la hegemonía se entiende como un liderazgo internacional, particularmente en el ámbito de la economía política mundial. Este concepto de hegemonía se reserva fundamentalmente para definir un tipo de orden político-económico bajo el liderazgo de un hegemón (DANNER; MARTíN, 2019), y se diferencia de la noción de colonialismo, imperio, imperialismo u otro tipo de gobierno mundial.

La teoría de la hegemonía asociada enfatiza en el papel de un Estado hegemónico en la creación de régimen monetario internacional y liberalización del comercio (YARBROUGH; YARBROUGH, 1985). Aunque la política de libre comercio no es prioridad, ni la primera política del Estado hegemónico. En ese sentido, respecto a la política de comercio internacional, la TEH intenta explicar los cambios en la apertura o restricciones del comercio y su nivel relativo de intercambio (SACHSE, 1989).

De esta manera, destacan Gran Bretaña y Estados Unidos que, en sus periodos de preeminencia respectivamente, parecen encajar 
perfectamente en la noción de Estado Hegemónico y actuaron, por lo menos en ciertos momentos, para crear un sistema abierto que emergió durante el período de preeminencia (MCKEOWN, 1983).

A finales del siglo XIX y principios del siglo XX, Gran Bretaña era considerada una potencia comercial de alcance global con interés en favorecer la liberalización comercial bajo la premisa de beneficios para la sociedad y de los demás países (VAN DE HAAR, 2009). Por otro lado, Estados Unidos en el siglo XX, toma la iniciativa de establecer instituciones internacionales como el Fondo Monetario Internacional (FMI), el Banco Mundial (BM), el Acuerdo General sobre Aranceles Aduaneros y Comercio (GATT), y la Organización para la Cooperación y el Desarrollo Económico (OCDE) con el objetivo de consolidar una economía mundial liberal reformada (JACKSON; SØRENSEN, 2016)

Estados Unidos ha desempeñado el papel de Estado Hegemónico en la configuración del orden económico global. Sin embargo, a inicios del siglo XXI ha experimentado una serie de sucesos que han hecho que su poder entre en crisis. Sucesos como la división transatlántica a principios de la década de 2000, durante la Guerra de Irak, y la Crisis Financiera Global de 2008, en la cual la confianza se desmoronó en la capacidad para liderar el orden económico global (LEHTI; PENNANEN; JOUHKI, 2019). Es por ello que con el auge económico de China que se ha transformado en una economía pujante y con múltiples inversiones en muchos países del mundo, políticas económicas poco ortodoxas han dado paso al inicio de un nuevo orden.

La presencia internacional de China se centra únicamente en las preocupaciones comerciales y financieras, en donde asume pacíficamente y de manera gradual el liderazgo internacional que ocupa Estados Unidos. Para ello promoverá el comercio en un sistema internacional que excluye cualquier forma de conflictos violentos o imposición de normas e ideologías políticas, motivado principalmente por intenciones económicas 
neutrales centradas en el Estado, por alcanzar objetivos puramente nacionalistas y por intereses propios, muy al estilo de los Países Bajos en los siglos XVI y XVII (DANNER; MARTÍN, 2019).

\section{EL ROL DE CHINA EN LA ECONOMÍA GLOBAL}

El crecimiento económico de China es un caso particular y, quizá, sin precedentes entre las economías emergentes. En tan solo 40 años se convirtió en la segunda economía más importante del mundo, y aporta más del 15\% del PIB mundial, sólo superada por Estados Unidos (Tabla 1). Entre el año 2000 y 2018, su economía tuvo un crecimiento promedio de 9,1\% (Figura 1), incluso en el periodo posterior a la Crisis Financiera Global de 2008, esto refleja una consolidación de su economía. Además, la contribución en la producción global de China incrementó en un 26\%, cifra superior a la contribución de la Zona Euro y los Estados Unidos combinados (OXFORD ECONOMICS, 2017). Así, su mercado se ha consolidado como uno de los principales destinos de exportaciones e importaciones, además de un lugar fundamental para la consecución de negocios internacionales por su presencia en las cadenas productivas globales (URIBE, 2010).

Tabla 1 - Porcentaje del PIB respecto al total global (porcentaje)

\begin{tabular}{lrrrrrrr}
\hline País & $\mathbf{1 9 7 0}$ & $\mathbf{1 9 8 0}$ & $\mathbf{1 9 9 0}$ & $\mathbf{2 0 0 0}$ & $\mathbf{2 0 1 0}$ & $\mathbf{2 0 1 5}$ & $\mathbf{2 0 1 8}$ \\
\hline Estados Unidos & 31,7 & 23,3 & 26,1 & 30,7 & 22,8 & 24,5 & 24,1 \\
China & 2,7 & 2,5 & 1,7 & 3,6 & 9,2 & 14,8 & 15,9 \\
Japón & 6,2 & 9,0 & 13,6 & 14,6 & 8,6 & 5,9 & 5,8 \\
Alemania & 6,3 & 7,7 & 7,7 & 5,8 & 5,2 & 4,5 & 4,7 \\
Reino Unido & 3,8 & 4,6 & 4,8 & 4,9 & 3,7 & 3,9 & 3,3 \\
Francia & 4,4 & 5,7 & 5,5 & 4,1 & 4,0 & 3,3 & 3,3 \\
India & 1,8 & 1,5 & 1,4 & 1,4 & 2,5 & 2,9 & 3,2 \\
Brasil & 1,0 & 1,6 & 1,8 & 1,9 & 3,3 & 2,4 & 2,2 \\
Italia & 3,3 & 3,9 & 5,1 & 3,4 & 3,2 & 2,5 & 2,4 \\
Canadá & 2,6 & 2,2 & 2,6 & 2,2 & 2,4 & 2,1 & 2,0 \\
Colombia & 0,3 & 0,4 & 0,2 & 0,3 & 0,4 & 0,4 & 0,4 \\
Resto & 35,8 & 37,7 & 29,5 & 27,1 & 34,5 & 32,9 & 32,6 \\
\hline
\end{tabular}

Fuente: Elaboración propia a partir de los datos de UNCTAD (2020a). 
Este crecimiento económico de magnitudes inimaginables es gracias a las políticas poco ortodoxas que han llevado a una transformación económica. Las políticas se enfocaron al fortalecimiento del sector agrícola para maximizar el uso de la tierra y destinar toda la tierra cultivable para la alimentación de la población. También, en el cambio de la estructura productiva a pesar de la dualidad económica en sus sectores, se otorgaron más libertades a los empresarios y se hizo hincapié en la producción de bienes de consumo. Del mismo modo, las políticas se orientaron al aumento del grado de especialización del comercio internacional y apoyado en un fuerza laboral, para introducir técnicas modernas de producción guiadas por la inversión pública en sectores estratégicos y complementarios a la inversión privada con el fin de promover sus exportaciones en el mercado global (ROMERO; LÓPEZ; ESCOBAR, 2017), planteando un modelo de desarrollo económico con similitudes a los propuestos por la corriente neoestructuralista (BHADURI, 2003; TAYLOR, 1990).

Figura 1 - Crecimiento económico 2000-2018

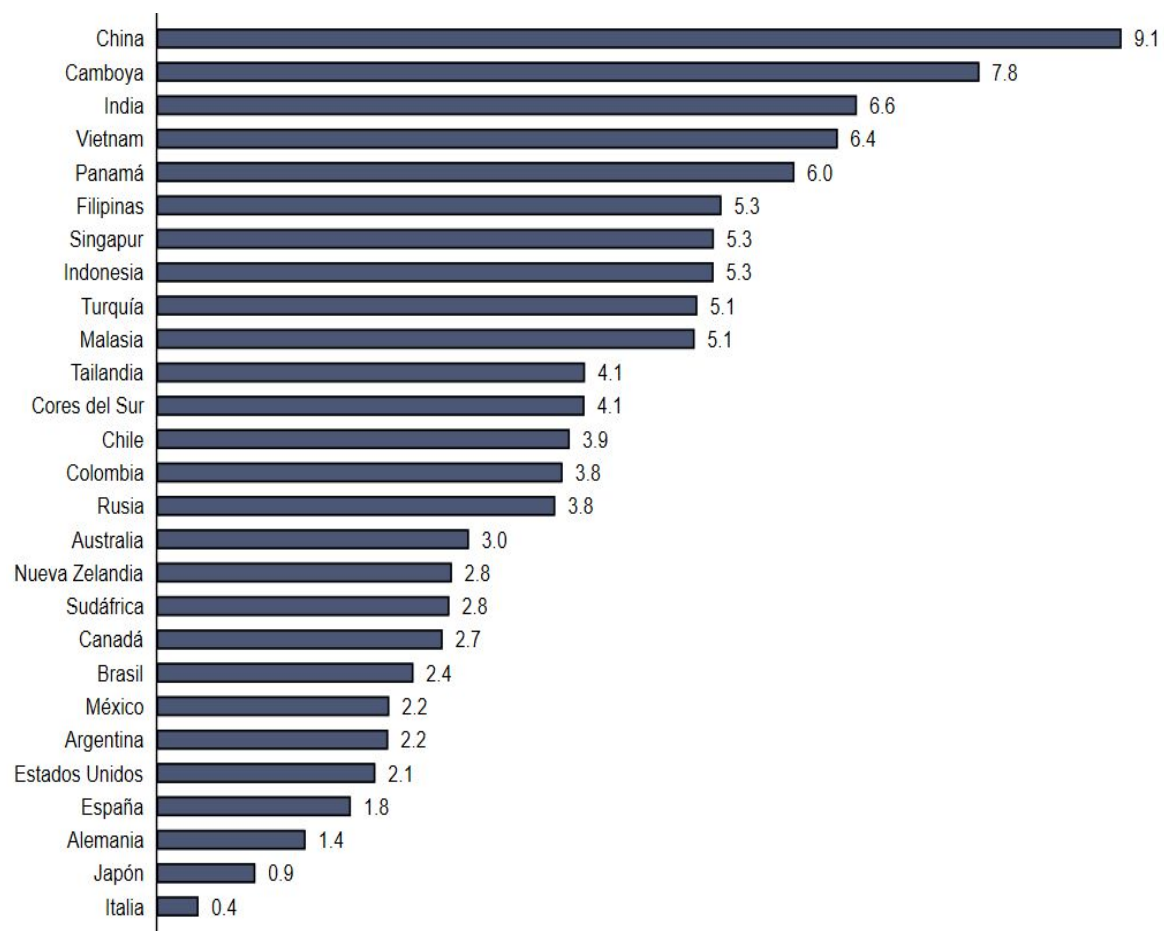

Fuente: Elaboración propia a partir de datos del Banco Mundial (2020). 
El tamaño de la economía en diversos sectores económicos y el nivel de desarrollo económico han hecho que China surja como una potencia económica a nivel global. Además de los altos niveles de crecimiento económico, la participación en comercio internacional se ha incrementado de manera significativa, lo que ha llevado a esta economía a una posición dominante en el Comercio global. Desde el año 2009 ha sido el mayor exportador de mercancías del mundo y el segundo mayor importador, siendo superado solo por Estados Unidos, posicionándose como la economía con mayor participación en el comercio internacional (Tabla 2). Lo anterior, en parte, ha sido gracias al ingreso de China en la Organización Mundial del Comercio (OMS) en el año 2001, lo que llevó a la nación a un periodo mayor de liberalización comercial en el que se le otorgó más poder a intereses de empresas privadas (BORDEN, 2012).

Tabla 2 - Participación en el comercio internacional ${ }^{3}$ (porcentaje)

\begin{tabular}{lrrrrrrr}
\hline País & $\mathbf{1 9 7 0}$ & $\mathbf{1 9 8 0}$ & $\mathbf{1 9 9 0}$ & $\mathbf{2 0 0 0}$ & $\mathbf{2 0 1 0}$ & $\mathbf{2 0 1 5}$ & $\mathbf{2 0 1 8}$ \\
\hline China & 0,71 & 0,92 & 1,62 & 3,62 & 9,68 & 11,9 & 11,8 \\
Estado Unidos & 13,2 & 11,7 & 12,8 & 15,6 & 10,6 & 11,5 & 10,9 \\
Alemania & - & - & 10,9 & 7,98 & 7,53 & 7,14 & 7,24 \\
Japón & 5,89 & 6,56 & 7,36 & 6,55 & 4,76 & 3,83 & 3,78 \\
Holanda & 4,48 & 4,19 & 3,63 & 3,44 & 3,55 & 3,25 & 3,49 \\
Francia & 5,83 & 6,13 & 6,45 & 5,07 & 3,69 & 3,24 & 3,19 \\
Reino Unido & 6,37 & 5,45 & 5,76 & 4,82 & 3,28 & 3,29 & 2,95 \\
República de & & & & & & & \\
Corea & 0,43 & 0,96 & 1,9 & 2,54 & 2,9 & 2,89 & 2,9 \\
Italia & 4,34 & 4,32 & 4,96 & 3,66 & 3,04 & 2,61 & 2,68 \\
México & 0,6 & 0,97 & 1,19 & 2,64 & 1,98 & 2,36 & 2,36 \\
Colombia & 0,24 & 0,21 & 0,17 & 0,19 & 0,26 & 0,27 & 0,24 \\
\hline
\end{tabular}

Fuente: Elaboración propia a partir de los datos de UNCTAD (2020b).

El éxito económico de China ha beneficiado a otras economías a través de diferentes canales. El aumento de las importaciones del país asiático ha tenido un impacto positivo en sus socios comerciales en términos de sus exportaciones y PIB. Por otro lado, el aumento de las exportaciones de China tiene un impacto positivo por los bajos costos relativos de los productos, favoreciendo el consumo y la producción de los

\footnotetext{
${ }^{3}$ Se calcula como la suma de las exportaciones e importaciones de cada país dividido entre la suma del total de exportaciones $y$ el total de importaciones a nivel mundial. $P C=X i+M i / X+M$
} 
socios comerciales (ARORA; VAMVAKIDIS, 2011). Por tal motivo, el rol de China en la globalización es cada vez más relevante por su presencia en las cadenas globales de valor, al demandar insumos y materias primas y ser una economía exportadora de bienes manufacturados al resto del mundo. Economías del continente asiático, como Singapur, Corea del Sur, Hong Kong y Taiwán, son las que más se han beneficiado del rápido crecimiento económico de China por el encadenamiento productivo entre esas economías, sirviendo de proveedores de insumos y capital intensivo para así impulsar el crecimiento y desarrollo de las economías de la región.

En América Latina, Colombia presenta un crecimiento promedio superior a los de muchos países de la región, a pesar de la CFG de 2008. Las exportaciones de Colombia hacia China han incrementado desde el año 2002, aunque los incrementos son mayores a partir del 2008, hasta el punto de concentrar alrededor del 10\% del total de las exportaciones, convirtiéndose así en un mercado fundamental para los exportadores colombianos. Debido al plan de infraestructura impulsado por el gobierno chino, la composición de las exportaciones colombianas hacia ese país se modificó, pasando de exportar productos industriales a mediados de la década de los años 90, hasta inicio de los años 2000, a la exportación de productos primarios principalmente (Figura 2). 
Figura 2 - Total exportaciones de Colombia hacia China, 1995-2018
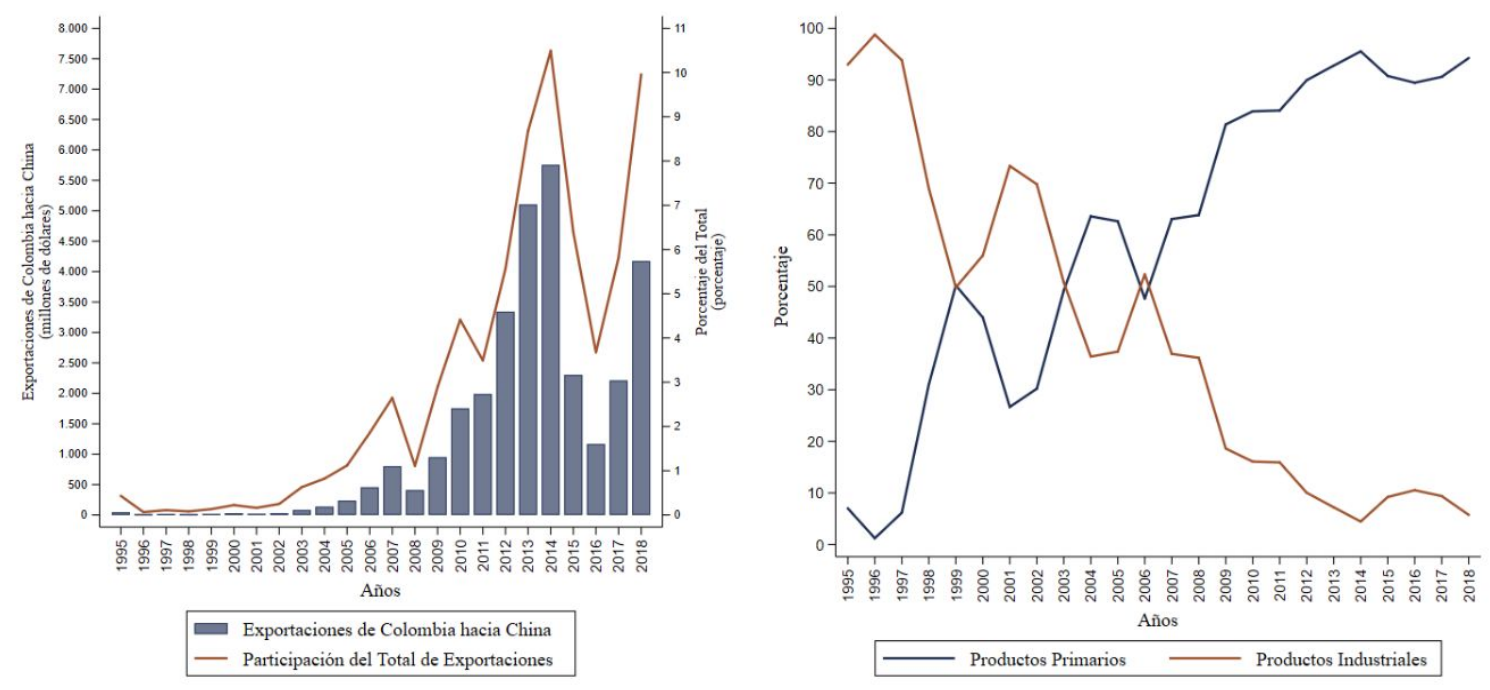

Fuente: Elaboración propia a partir de los de DIAN-DANE (2019).

\section{COMERCIO INTERNACIONAL ENTRE CHINA Y COLOMBIA}

Desde hace décadas, Estados Unidos ha sido el principal destino de las exportaciones colombianas, así como el principal proveedor de bienes y de servicios, siendo el principal socio comercial de Colombia. China, por su parte, ha llegado a ocupar una posición importante como socio comercial, siendo superado solo por los Estados Unidos (Figura 3). Se destaca que los vínculos comerciales entre Colombia y China se han incrementado notablemente desde hace más de dos décadas. Ese fortalecimiento de los vínculos y el aumento del flujo comercial con China se debe a la necesidad de diversificar el portafolio comercial de Colombia para promover las exportaciones $\mathrm{y}$, posiblemente, hacer frente cualquier choque en la economía global. Sin embargo, Colombia está rezagada, en comparación con otros países de la región. En cuanto a las relaciones con China, se ha postergado la densificación de los lazos debido a la relación especial entre Bogotá y Washington (BORDA GUZMÁN; BERGER, 2012; DE LA HOZ et al., 2014; ESCOBAR ESPINOZA, 2012; ESCOBAR, 2009). 
Figura 3 - Comercio entre Colombia y principales países, 1990-2018
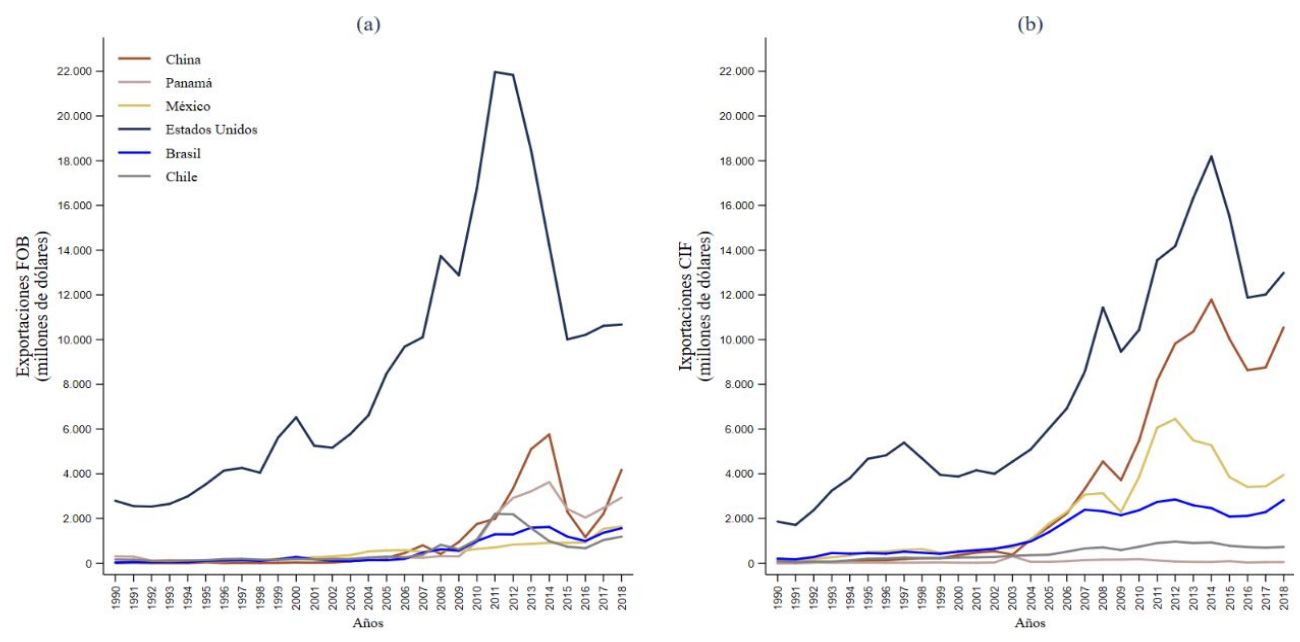

Fuente: Elaboración propia a partir de datos de DIAN-DANE (2020a, 2020b).

Aunque las exportaciones colombianas hacia China han incrementado notablemente, el portafolio de exportaciones no es diversificado, es decir, se encuentra concentrado en sectores determinados (Figura 4). El sector de suministros industriales tuvo la mayor participación de las exportaciones hacia China alcanzando en 2007, y previo a la CFG, el 98\% del total; con esto, el sector industrial colombiano se integra poco a poco en las cadenas de suministros globales (ESCOBAR, 2016). Posteriormente, se incrementan de manera acelerada las exportaciones de materia prima, en particular el sector de petróleo, que en 2014 alcanzó su máximo de participación con el 92\% del total de exportaciones. 
Figura 4 - Exportaciones de Colombia hacia China, 2000-2018 (clasificación BEC)

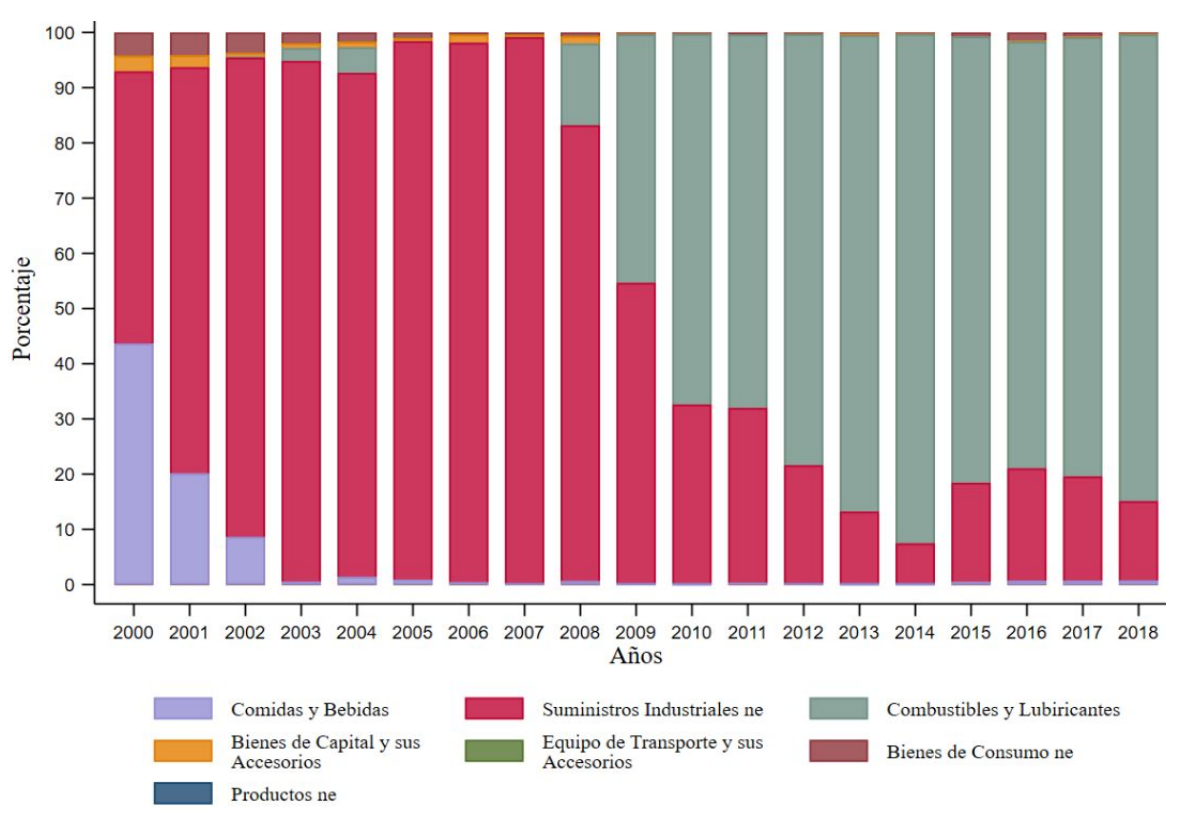

Fuente: Elaboración propia a partir de datos de COMTRADE (2020).

Los sectores exportadores de Colombia cada vez se integran de manera acelerada con China. En el año 2000, se exportaron alrededor de USD 30 millones, alcanzando un máximo en 2014 con cerca de USD 5.700 millones y, finalmente, en el 2018 se exportaron más de USD 3.900 millones. Esto demuestra una mayor importancia del mercado de Asia para Colombia. Por otro lado, seguido del petróleo y gas, el comercio al por mayor y la metálica básica de hierro y acero lideran las principales exportaciones hacia China (Tabla 3), con una variación porcentual del $95,4 \%, 86,8 \%$ y $25,2 \%$ respectivamente. 
Tabla 3 - Principales exportaciones colombianas hacia China (millones de USD y variación porcentual)

\begin{tabular}{cccccc}
\hline $\begin{array}{c}\text { Código } \\
\text { CIIU }\end{array}$ & Descripción & $\mathbf{2 0 1 6}$ & $\mathbf{2 0 1 7}$ & $\mathbf{2 0 1 8}$ & $\begin{array}{c}\text { Variación } \\
\mathbf{2 0 1 7 - 2 0 1 8}\end{array}$ \\
\hline 220 & Petróleo y gas & $1.117,88$ & $1.802,68$ & $3.523,23$ & 95,4 \\
371 & Metálicas básicas de hierro y acero & 86,10 & 159,36 & 297,76 & 86,8 \\
610 & Comercio al por mayor & 89,99 & 139,76 & 175,04 & 25,2 \\
354 & Derivados del petróleo & 6,17 & 21,00 & 53,62 & 155,3 \\
311 & Fabricación de productos & 14,26 & 18,40 & 27,46 & 49,2 \\
& alimenticios & & & & \\
323 & Cuero y sus derivados & 16,20 & 24,09 & 11,30 & $-53,1$ \\
351 & Químicos industriales & 15,46 & 13,98 & 9,99 & $-28,5$ \\
210 & Extracción de minas de carbón & 0,0008 & 0 & 20,12 & - \\
230 & Extracción de minerales metálicos & 10,41 & 6,10 & 15,46 & 153,3 \\
352 & Otros químicos & 2,72 & 7,01 & 9,31 & 32,8 \\
122 & Extracción de madera & 3,39 & 2,62 & 6,81 & 159,6 \\
\hline
\end{tabular}

Fuente: Elaboración propia a partir de datos del Sistema Estadístico de Comercio Exterior (2020).

El valor de las importaciones provenientes de China viene en aumento continúo desde el año 2003. En el 2018 el valor de esas importaciones supera los USD 10.000 millones, alrededor de 27 veces más que el año 2003. Los principales productos de importación son los bienes de capital e insumos para la producción del sector industrial, los primeros se mantienen como las importaciones con mayor participación (Figura 5). En consecuencia, el aumento de las importaciones ha beneficiado especialmente a los empresarios de Colombia, dado que tienen acceso a maquinarias y equipos necesarios para llevar a cabo los procesos productivos (Tabla 4). 
Figura 5 - Importaciones de Colombia provenientes de China, 2000-2018 (clasificación BEC)

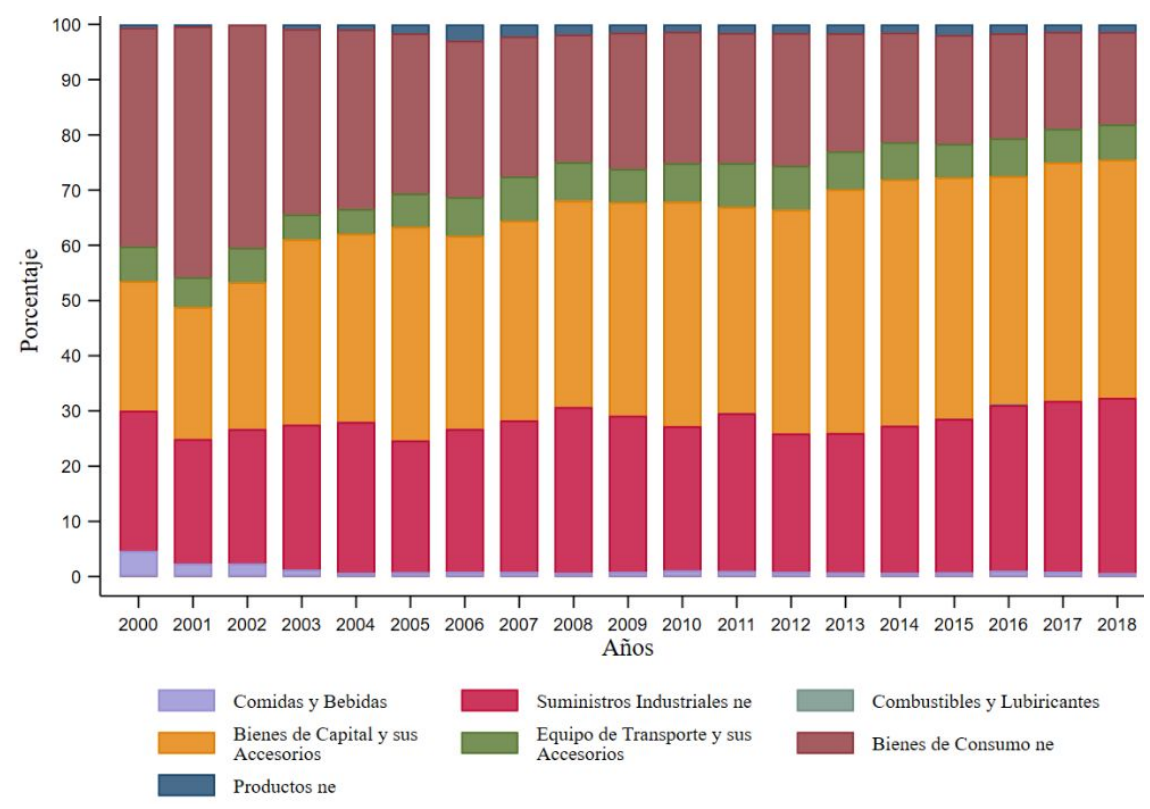

Fuente: Elaboración propia a partir de datos de COMTRADE (2020).

Tabla 4- Principales importaciones colombianas provenientes de China (millones de USD y variación porcentual)

\begin{tabular}{|c|c|c|c|c|c|}
\hline $\begin{array}{l}\text { Código } \\
\text { CIIU }\end{array}$ & Descripción & 2016 & 2017 & 2018 & $\begin{array}{c}\text { Variació } \\
n \\
2017-201 \\
8\end{array}$ \\
\hline 383 & Maquinaria eléctrica & $2.303,06$ & $2.492,29$ & $3.157,47$ & 26,7 \\
\hline 382 & Maquinaria excluida la eléctrica & $1.435,96$ & $1.369,40$ & $1.523,37$ & 11,2 \\
\hline 321 & Textiles & 621,94 & 608,20 & 818,44 & 34,6 \\
\hline 351 & Químicos industriales & 670,65 & 638,93 & 672,46 & 5,2 \\
\hline 384 & Material de transporte & 494,31 & 421,00 & 534,91 & 27,1 \\
\hline 381 & $\begin{array}{c}\text { Metalmecánica excluida } \\
\text { maquinaria }\end{array}$ & 437,57 & 423,81 & 502,20 & 18,5 \\
\hline 371 & Metálicas básicas de hierro y acero & 275,51 & 345,18 & 535,55 & 55,2 \\
\hline 356 & Plásticos & 293,45 & 312,23 & 373,20 & 19,5 \\
\hline 390 & Otras industrias manufactureras & 287,42 & 303,43 & 338,43 & 11,5 \\
\hline 385 & Equipo profesional y científico & 213,25 & 207,70 & 242,01 & 16,5 \\
\hline 352 & Otros químicos & 182,67 & 188,73 & 228,83 & 21,2 \\
\hline 355 & Caucho & 181,46 & 173,65 & 216,11 & 24,5 \\
\hline 322 & Prendas de vestir & 148,12 & 162,22 & 179,40 & 10,6 \\
\hline 323 & Cuero y sus derivados & 105,86 & 116,60 & 130,53 & 11,9 \\
\hline
\end{tabular}

Fuente: Elaboración propia a partir de datos del Sistema Estadístico de Comercio Exterior (2020).

Si bien es cierto que el flujo comercial entre Colombia y China ha aumentado de manera significativa, del mismo modo lo ha hecho la brecha entre las exportaciones e importaciones. Aunque el valor de las exportaciones de Colombia hacia China ha crecido durante los últimos años, el valor de las importaciones proveniente de China ha crecido de 
manera más acelerada generando un déficit comercial para Colombia (Figura 6), con probabilidad de generar impactos negativos a nivel sectorial y regional, con posibles repercusiones sociales y económicas (GARCÍA-GARCÍA, 2017). No obstante, se incurre al costo de sacrificar producción de bienes nacionales para el desarrollo y la consolidación de las relaciones bilaterales con China, que en el mediano o largo plazo puede beneficiar a la economía colombiana (GÓMEZ; ESCOBAR, 2016).

Figura 6 - Comercio de Colombia con China, 2000 - 2018

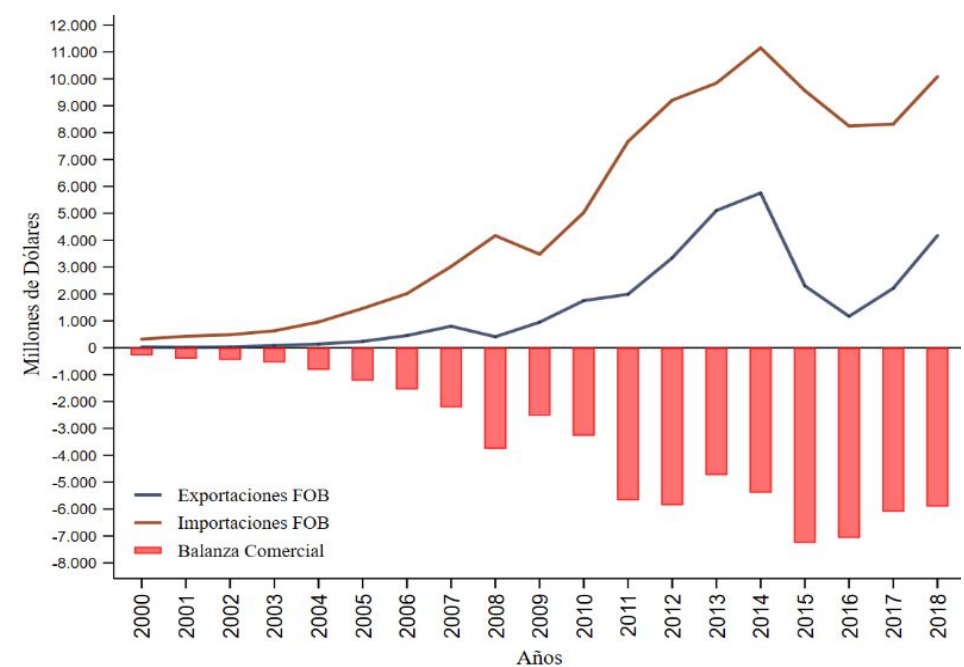

Fuente: Elaboración propia a partir de datos de DIAN-DANE (2020c).

En virtud de la dotación factorial de las economías emergentes, estas se especializan en la exportación de productos primarios. Tal es el caso de Colombia, donde la mayor proporción de sus exportaciones se concentran en petróleo y gas. Por otro lado, China se especializa en bienes industriales con mayor valor agregado. La partición de la manufactura en las exportaciones de mercancías de China incrementó de 72\% a 93\% entre 1990 y 2007, manteniéndose en esos niveles desde entonces. Esta concentración comercial muestra que el crecimiento de China ha tenido efectos positivos tanto para la oferta mundial de manufacturas como para la demanda mundial de materia primas (AUTOR; DORN; HANSON, 2015).

Hoy en día, se discute si China es socialista o capitalista por la serie de reformas ortodoxas que se han implementado con un rol protagónico del Estado, lo cual comúnmente denominan algunos como socialismo de 
mercado con particularidades chinas, que de momento ha sido una combinación efectiva entre los dos sistemas. En 1980, se crearon cinco Zonas Económicas Especiales (ZEE) que, bajo el control administrativo del gobierno de China, brindan una serie de beneficios fiscales a los inversionistas y garantizan una alta rentabilidad debido a la flexibilización de las regulaciones. Por ello, se estimuló la inversión extranjera. Sobre todo, con las multinacionales de uso intensivo de capital que servirían como base de aprendizaje de las empresas chinas, lo que ha permitido que China se convirtiera en uno de los destinos preferentes para la IED mundial (PIÑA, 2015).

Para el caso de Colombia, sus inversiones en China generan ingresos para el sector privado y se convierten cada vez más en atractivos para los inversionistas colombianos. Previo a la CGF de 2008, los volúmenes de Inversión de Colombia en el Exterior (IDCE) en China eran superiores que en los años posteriores (Figura 5). Sin embargo, el flujo de IED que ingresa a Colombia proveniente de China no ha sido consistente a lo largo del tiempo y, además, es limitado, no supera el 1\% del total de IED china (CASTAÑEDA, 2017). En cuanto a la inversión en activos financieros, Colombia es el tercer país de América Latina con mayor inversión en cartera por parte de China (Tabla 5), de la cual la mayor proporción la representan las inversiones en bonos de deuda. 
Tabla 5 - Activos de inversión de cartera externa de China en América Latina (millones de USD)

\begin{tabular}{lrrc}
\hline \multicolumn{1}{c}{ País } & dic-18 & dic-19 & Variación \\
\hline Brasil & $1.638,35$ & $1.876,14$ & $14,5 \%$ \\
México & 871,78 & 987,16 & $13,2 \%$ \\
Colombia & 201,60 & 223,18 & $10,7 \%$ \\
Chile & 196,09 & 194,53 & $-0,8 \%$ \\
Perú & 147,08 & 177,94 & $21,0 \%$ \\
Panamá & 87,83 & 123,05 & $40,1 \%$ \\
Ecuador & 78,91 & 102,70 & $30,1 \%$ \\
Argentina & 234,42 & 99,99 & $-57,3 \%$ \\
República & & & \\
Dominicana & 94,45 & 85,63 & $-9,3 \%$ \\
Resto América Latina & 269 & 255,58 & $-4,99 \%$ \\
\hline
\end{tabular}

Fuente: SAFE (2020).

Figura 5 - Inversión Directa de Colombia en China (millones de USD)

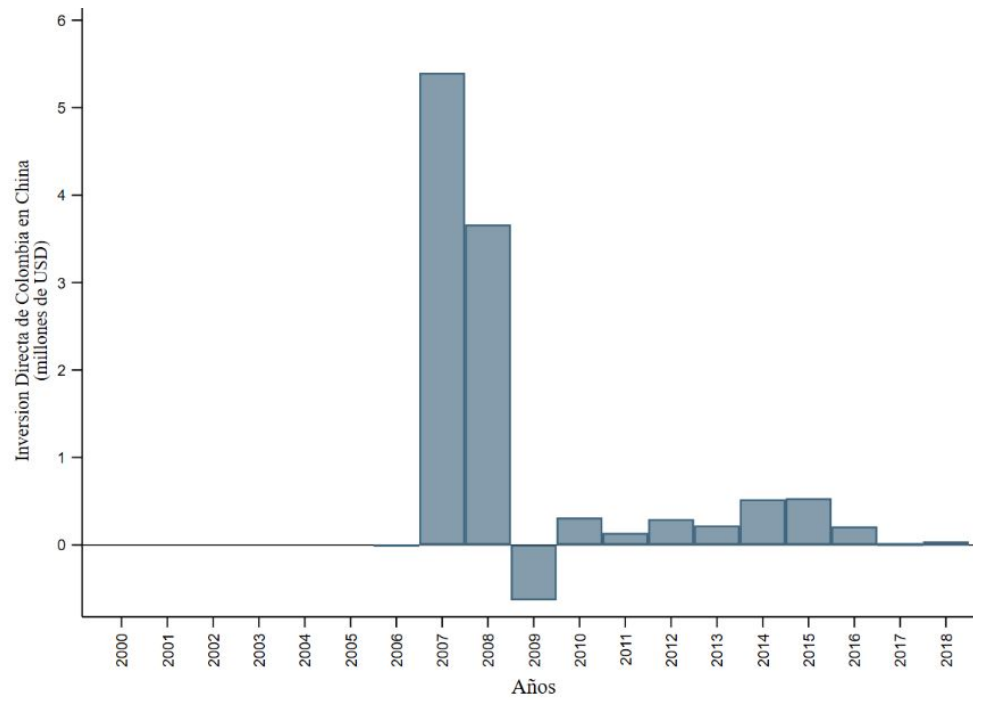

Fuente: Elaboración propia a partir de datos del Banco de la República (2020). Nota: Un flujo negativo significa que las liquidaciones de inversiones directas en el exterior son mayores a las constituciones.

\section{BENEFICIOS DE LA INTEGRACIÓN ECONÓMICA CON CHINA}

Desde los primeros desarrollos de la teoría de ventajas comparativas, las naciones presentan ganancias del comercio internacional a través de la especialización en los bienes que producen a costos relativos menores (KRUGMAN; OBSTFELD; MELITZ, 2018). El comercio internacional y la especialización productiva generan cambios en las estructuras económicas de las naciones, y también en los salarios y rentas de los factores 
productivos utilizados en la producción de bienes y servicios. Por tanto, a pesar de los beneficios generales que puede traer consigo el comercio internacional, también puede generar ganadores y perdedores. Por lo anterior, existen muchos debates al interior de las economías en cómo avanzar o profundizar las políticas de liberación comercial.

Con el fin de analizar los aspectos inherentes a la implementación de políticas comerciales, que incluye la liberación comercial unilateral, reformas multilaterales a través de la Organización Mundial del Comercio, y acuerdos preferenciales de comercio internacional, se han empleado técnicas de modelación macroeconómica mediante la construcción de Modelos de Equilibrio General Computable (MEGC). Los aportes de estos análisis a partir del uso de esta metodología se enfocan en su habilidad de identificar la expansión o contracción de sectores económicos como consecuencia del libre comercio, también permiten describir el comportamiento de los factores productivos como trabajo y capital, y sobre todo medir los efectos en el bienestar (BURFISHER, 2017; FLORES-TAPIA; FLORES-CEVALLOS, 2017; ESCOBAR, 2016; MISSAGLIA, 2015; ESCOBAR; GUEVARA; URIBE, 2017).

Respecto a las tarifas arancelarias entre ambos, se destaca Colombia con mayores aranceles a las importaciones de bienes chinos (Tabla 6). Los aranceles impuestos por Colombia a la entrada de bienes agrícolas provenientes de China supera considerablemente a la tarifa impuesta por China a la entrada de productos agrícolas provenientes de Colombia. De la misma manera sucede con los aranceles a las importaciones de manufacturas. Los niveles de aranceles impuestos a China superan, incluso, al promedio de aranceles impuesto al resto del mundo. 
Tabla 6 - Aranceles promedio impuestos por China y Colombia al comercio internacional bilateral (porcentaje)

\begin{tabular}{|c|c|c|c|c|}
\hline \multirow[b]{2}{*}{ Sector } & \multicolumn{2}{|c|}{ Aranceles de China } & \multicolumn{2}{|c|}{ Aranceles de Colombia } \\
\hline & $\begin{array}{l}\text { Bienes } \\
\text { provenientes } \\
\text { de Colombia }\end{array}$ & $\begin{array}{c}\text { Bienes } \\
\text { provenientes } \\
\text { del Resto del } \\
\text { Mundo }\end{array}$ & $\begin{array}{l}\text { Bienes } \\
\text { provenientes } \\
\text { de China }\end{array}$ & $\begin{array}{c}\text { Bienes } \\
\text { provenientes } \\
\text { del Resto del } \\
\text { Mundo }\end{array}$ \\
\hline Agricultura & 5,25 & 4,91 & 17,68 & 13,2 \\
\hline Manufacturas & 1,68 & 4,21 & 12,58 & 8,31 \\
\hline Servicios & 0 & 0 & 0 & 0 \\
\hline
\end{tabular}

Fuente: GTAP v.8.1 COL 3×3 database (AGUIAR; NARAYANAN; MCDOUGALL, 2016).

Una forma de analizar el impacto económico de la integración comercial bilateral es mediante la eliminación de los aranceles bilaterales y los posibles efectos en ambas economías. En este sentido, a partir del modelo global GTAP (HERTEL, 1996) se analizan los efectos de una eliminación total de aranceles por parte de China y Colombia, pero conservando los aranceles para el resto de países.

Si se considera la balanza comercial por sectores económicos, presenta un efecto favorable para Colombia en los sectores de agricultura y servicios (Tabla 7). Sin embargo, la posición de China mejora para el caso del sector industrial siendo consecuente con desarrollos en ese sector del país asiático.

Tabla 7- Eliminación de aranceles: Balanza comercial (X-M) (millones de USD)

\begin{tabular}{|c|c|c|}
\hline Sector & China & Colombia \\
\hline Agricultura & $-18,25$ & 13,66 \\
\hline Manufactura & 287,77 & $-169,33$ \\
\hline Servicios & $-157,29$ & 48,66 \\
\hline
\end{tabular}

Fuente: GTAP v.8.1 COL 3×3 database (AGUIAR; NARAYANAN; MCDOUGALL, 2016).

Es importante considerar el cambio en los volúmenes del comercio entre ambos países como consecuencia de una mayor integración económica con el fin de identificar los mecanismos a nivel sectorial de la eliminación completa de aranceles. De esta manera, se muestra que un acuerdo comercial favorece la creación de comercio bilateral dado que, para ambas economías, el volumen bilateral de comercio se incrementa 
por encima de aquellos que se encuentran fuera del acuerdo, en particular para los sectores de agricultura e industria (Tabla 8). Por otro lado, un acuerdo comercial bilateral tendría más efectos en la estructura productiva de Colombia, en particular en el sector de Agricultura (Tabla 9) que presenta mayores cambios con respecto al año base. Situación similar se presenta en la estructura de las exportaciones colombianas con el resto del mundo, estimulando las exportaciones no solo del sector agrícola sino sobre todo aquellas del sector industrial. Lo anterior da cuenta de los encadenamientos productivos al interior de la economía, comparado con otros sectores, favoreciendo la producción de bienes intermedios. Si bien es cierto que se presenta una creación de comercio internacional entre ambos países, sus efectos en términos de estructura productiva y de canasta exportadora para el caso de China son mínimos.

Tabla 8- Eliminación de aranceles: Volúmenes de comercio internacional

\begin{tabular}{|c|c|c|c|c|}
\hline Sector & $\begin{array}{c}\text { Importaciones a } \\
\text { China desde } \\
\text { Colombia (millones } \\
\text { de USD) }\end{array}$ & $\begin{array}{c}\text { Importaciones } \\
\text { a China desde } \\
\text { Colombia (\%) }\end{array}$ & $\begin{array}{c}\text { Importaciones a } \\
\text { Colombia desde } \\
\text { China } \\
\text { (millones de USD) }\end{array}$ & $\begin{array}{c}\text { Importaciones } \\
\text { a Colombia } \\
\text { desde China } \\
(\%)\end{array}$ \\
\hline Agricultura & 1,03 & $28,82 \%$ & 21,47 & $115,62 \%$ \\
\hline Manufactura & 158,44 & $16,73 \%$ & 2971,93 & $121,49 \%$ \\
\hline Servicios & 1,07 & $1,10 \%$ & $-0,89$ & $-0,65 \%$ \\
\hline
\end{tabular}




\begin{tabular}{lccc}
\hline \multicolumn{2}{c}{ Variable } & Colombia & China \\
\hline Producción & Agricultura & 0,11 & $-0,002$ \\
& Industrial & 0,01 & 0,0001 \\
Exportaciones & Servicios & $-0,01$ & 0,0006 \\
& & & \\
& Agricultura & 0,72 & 0,02 \\
Industrial & 3,29 & 0,067 \\
Producción intermedia & 0,95 & $-0,10$ \\
& Servicios & & \\
& Agricultura & 0,11 & $-0,002$ \\
& Industrial & 0,012 & 0,0001 \\
& Servicios & $-0,01$ & 0,0006 \\
\hline Fuente: GTAP v.8.1 COL 3x3 database (AGUIAR; NARAYANAN; MCDOUGALL, 2016).
\end{tabular}

\section{CONCLUSIONES}

La economía china y su desempeño, junto con una mayor integración comercial con Colombia, se constituyen como elementos importantes para el desarrollo económico colombiano. La situación persistente de la débil demanda global como consecuencia de la Crisis Financiera Global del 2008 y sumados, hoy en día, a los efectos adversos en los mercados ocasionados por la pandemia causada por el SARS-CoV-2 (COVID-19), indican que se requieren aprovechar al máximo las sinergias y los beneficios de una mayor integración económica internacional.

Es sabido que para el análisis de las políticas de comercio internacional se requiere un entendimiento que vaya más allá de los fundamentos de las ventajas comparativas, dado que existen razones distintas a las ganancias del comercio por las que dos economías pueden comerciar entre sí. Una de las razones son los intereses propios nacionales, motivados principalmente por preocupaciones financieras, que buscan solo maximizar beneficios. Tal como China que ha registrado crecimiento económico y financiero considerable e interés por países como Colombia, con abundancia en combustibles fósiles y minerales. Por tal motivo, 
Colombia debe garantizar recursos necesarios para continuar fortaleciendo su desarrollo económico y expandir el mercado de sus productos que presentan un mayor valor agregado.

A pesar de lo anterior, las relaciones económicas con China le permitieron a Colombia incrementar sus exportaciones y las importaciones de bienes de capital necesarios para incrementar la productividad. Las oportunidades y retos actuales, se encuentran en permitir que productos de mayor valor agregado cuenten con acceso al mercado asiático en virtud del mejoramiento de su poder adquisitivo.

De la misma manera, la profundización de la integración económica requiere incrementos en la productividad del trabajo en Colombia para poder ser competitivo en los mercados internacionales. Los beneficios de una mayor integración comercial con China se concentran en los incrementos en los volúmenes de exportaciones e importaciones, pero sobre todo en la diversificación de las exportaciones colombianas, favoreciendo la expansión de sectores como la industria y agricultura. Desafortunadamente, muchos de los beneficios de la profundización en las relaciones comerciales se ven opacados por sus costos, dejándose a un lado las ganancias netas del comercio. Los gobiernos nacionales deben hacer esfuerzos para la creación de programas que permitan a los trabajadores ser más productivos mediante la adquisición de competencias y destrezas necesarias en los sectores en expansión.

\section{REFERENCIAS}

AGUIAR, Angel.; NARAYANAN, Badri.; MCDOUGALL, Robert. An Overview of the GTAP 9 Data Base. Journal of Global Economic Analysis. v. 1, n. 1, p. 181-208, 2016. DOI: http://dx.doi.org/10.21642/JGEA.010103AF. Consultado: 9 agosto 2020 
ARORA, Vivek; VAMVAKIDIS, Athanasios. China's Economic Growth: International Spillovers. China \& World Economy, v. 19, n. 5, 2011. DOl: https://doi.org/10.1111/j.1749-124X.2011.01257.x. Consultado: 22 agosto 2020

AUTOR, David H.; DORN, David; HANSON, Gordon. H. The China Shock: Learning from Labor-Market Adjustment to Large Changes in Trade. Annual Review of Economics, v. 8, 2015. DOl: https://doi.org/10.1146/annurev-economics-080315-01504. Consultado: 27 agosto 2020

BANCO DE LA REPÚBLICA. Flujo de Inversión Directa de Colombia en el Exterior (IDCE). Inversión Directa. 2020. Disponible en: https://www.banrep.gov.co/es/estadisticas/inversion-directa. Consultado: 8 agosto 2020.

BANCO MUNDIAL. Crecimiento del PIB (\% anual). Datos de Libre Acceso del Banco $2020 . \quad$ Disponible en: https://datos.bancomundial.org/indicador/NY.GDP.MKTP.KD.ZG.

Consultado: 8 agosto 2020.

BHADURI, Amit. Structural Change and Economic Development: on the Relative Roles of Effective Demand and the Price Mechanism in a Dual Economy. En: Chang, H. J. (ed.), Rethinking Development Economics. London: Anthem Press, 2003, cap. 10.

BORDA GUZMÁN, Sandra; BERGER, María Paz. Relaciones bilaterales China y Colombia: 1990-2010. Colombia Internacional, n. 75, p. 83-129, 2012. Disponible en: https://revistas.uniandes.edu.co/doi/abs/10.7440/colombiaint75.2012.04. Consultado: 8 agosto 2020

BORDEN, Graham. China's Accession to the WTO: Economic Benefits. The Park Place Economist, v. 20, 2012. Disponible en: https://digitalcommons.iwu.edu/parkplace/vol20/isst/8.

\section{BURFISHER, Mary E. Introduction to Computable General Equilibrium}

Models. 2nd ed. Cambridge: Cambridge University Press, 2017. DOl: https://doi.org/10.1017/9781316450741. Consultado: 18 agosto 2020

CASTAÑEDA, Néstor. New Dependency?: Economic Links between China and Latin America. Issues \& Studies, v. 53, n. 1, 2017. DOI: https://doi.org/10.1142/S101325111740001X. Consultado: 19 agosto 2020

COMTRADE. International Trade Statistics Database. United Nations, 2020. Disponible en: https://comtrade.un.org/. Consultado: 8 agosto 2020. 
DANNER, Lukas K.; MARTÍN, Félix. E. China's hegemonic intentions and trajectory: Will it opt for benevolent, coercive, or Dutch-style hegemony. Asia \& the Pacific Policy Studies, v. 6, n. 2, 2019. DOl: https://doi.org/10.1002/app5.273. Consultado: 8 agosto 2020

DE LA HOZ, Andrea; CAMARGO MONTOYA, Paola; SALDARRIAGA ARANGO, Carolina; PRAJ, Dusán. Análisis de los riesgos en las importaciones colombianas. Panorama Económico, v. 22, n. 1, p. 63-75, 2014. DOI: https://doi.org/10.32997/2463-0470-vol.22-num.0-2014-1360. Consultado: 29 agosto 2020

DIAN-DANE. Exportaciones de Colombia Según Países de Destino. Comercio Internacional, 2020a. Disponible en: https://www.dane.gov.co/index.php/estadisticas-por-tema/comercio-intern acional/exportaciones. Consultado: 8 agosto 2020.

DIAN-DANE. Importaciones de Colombia Según Países de Origen. Comercio Internacional, 2020b. Disponible en: https://www.dane.gov.co/index.php/estadisticas-por-tema/comercio-intern acional/importaciones. Consultado: 8 agosto 2020.

DIAN-DANE. Balanza Comercial Según Grupos Económicos y Principales Países. Comercio Internacional, 2020c. Disponible en: https://www.dane.gov.co/index.php/estadisticas-por-tema/comercio-intern acional/balanza-comercial. Consultado: 8 agosto 2020.

DIAN-DANE. Estadísticas de Comercio Exterior de Colombia. Ministerio de Comercio, Industria y Turismo, 2019. Disponible en: https://www.mincit.gov.co/estudios-economicos/estadisticas-e-informes/co mercio-exterior-de-colombia. Consultado: 8 agosto 2020.

ESCOBAR ESPINOZA, Álvaro. Essays on Colombia 's economic development. 2012. Tesis (Doctorado en Política Económica), Università Cattolica del Sacro Cuore, Milano, 2012. Disponible en: http://hdl.handle.net/10280/1491. Consultado: 9 agosto 2020

ESCOBAR, Álvaro. El despertar de un tigre Andino: ¿Puede el crecimiento creciente de Colombia crear suficiente empleo?. Panorama Económico, v. 17, p. 154-191, 2009. Disponible en: https://revistas.unicartagena.edu.co/index.php/panoramaeconomico/article /view/371. Consultado: 9 agosto 2020

ESCOBAR, Álvaro. Stock-Flow consistent models for developing countries: The case of Colombia. En: 19th Annual Conference on Global Economic Analysis, 2016, Washington DC. Documento de Conferencia. Disponible en: 
https://www.gtap.agecon.purdue.edu/resources/res_display.asp?RecordID= 5102. Consultado: 8 agosto 2020

ESCOBAR, Álvaro.; GUEVARA, Diego Alejandro; URIBE, Miguel Andrés. Modelos Computacionales y Análisis de la Política Económica en Colombia. Panorama Económico, v. 25, n. 4, p. 535-558, 2017. DOl: https://doi.org/10.32997/2463-0470-vol.25-num.4-2017-2088. Consultado: 18 agosto 2020

FLORES-TAPIA, Carlos. E.; FLORES-CEVALLOS, Karla. L. Impactos económicos inducidos por la demanda final en sectores estratégicos para el cambio de la estructura productiva del Ecuador. Panorama Económico, v. 25, n. $3, \quad$ p. 443-458, $2017 . \quad$ DOI: https://doi.org/10.32997/2463-0470-vol.25-num.3-2017-2085. Consultado: 18 agosto 2020

GARCÍA-GARCÍA, Francisco. B. Debate sobre la inclusión del cooperativismo dentro de las políticas de la Nueva Ruralidad en América Latina. Panorama Económico, v. 25, n. 3, p. 357-380, 2017. DOI: https://doi.org/10.32997/2463-0470-vol.25-num.3-2017-208. Consultado: 19 agosto 2020

GÓMEZ, Kathy; ESCOBAR, Álvaro. Estructuralismo y desarrollo económico regional: contabilidad social en Cartagena de Indias. Revista Internacional de Cooperación y Desarrollo, v. 3, n. 2, p. 22-33, 2016. DOl: https://doi.org/10.21500/23825014.2777. Consultado: 19 agosto 2020

HERTEL, Thomas. W. Global Trade Analysis: Modeling and Applications. Cambridge: Cambridge University Press, 1996. DOI: https://doi.org/10.1017/CBO9781139174688. Consultado: 15 agosto 2020

JACKSON, Robert; SøRENSEN, Georg. Introduction to International Relations: Theories and Approaches. 6th ed. USA: Oxford University Press, 2016. DOI: https://doi.org/10.1093/hepl/9780198707554.001.0001. Consultado: 15 agosto 2020

JOSEPH, Jonathan. Hegemony. The Wiley-Blackwell Encyclopedia of Social Theory, p. $\quad$ p. $\quad 1-3, \quad 2017$ DOI: https://doi.org/10.1002/9781118430873.est0663. Consultado: 15 agosto 2020 KRUGMAN, Paul. R.; OBSTFELD, Maurice.; MELITZ, Marc. International Economics: Theory and Policy. 11th ed. Pearson, 2018.

LEHTI, Marko; PENNANEN, Henna-Riikka; JOUHKI, Jukka. Introduction. En: LEHTI, Marko; PENNANEN, Henna-Riikka; JOUHKI, Jukka. (ed.), Contestations of Liberal Order: The West in Crisis?. Palgrave Macmillan, 
2019, cap. 1. DOI: https://doi.org/10.1007/978-3-030-22059-4_1. Consultado: 16 agosto 2020

MCKEOWN, Timothy. J. Hegemonic stability theory and 19th century tariff levels in Europe. International Organization, v. 37, n. 1, p. 73-91, 1983. DOI: https://doi.org/10.1017/S0020818300004203. Consultado: 15 agosto 2020

MISSAGLIA, Marco. ¿Se pueden aplicar las ideas Keynesianas al largo plazo? Unas reflexiones teóricas y un modelo ilustrativo. Panorama Económico, v. 23, n. $\quad 1, \quad$ p. $\quad 17-30, \quad 2015 . \quad$ DOl: https://doi.org/10.32997/2463-0470-vol.23-num.1-2015-1380. Consultado: 18 agosto 2020

OXFORD ECONOMICS. Understanding the US-China Trade Relationship, 2017. Disponible

en:

https://www.uschina.org/reports/understanding-us-china-trade-relationshi p. Consultado: 17 agosto 2020

PIÑA, José Joaquín. Comercio exterior e inversión extranjera en China. Letras jurídicas: revista de los investigadores del Instituto de Investigaciones Jurídicas U. V., n. 32, p. 115-129, 2015. Disponible en: https://dialnet.unirioja.es/servlet/articulo?codigo $=5210667$. Consultado: 20 agosto 2020

ROMERO, Bernardo; LÓPEZ, Nicolás; ESCOBAR, Andrés. Breve análisis de las relaciones internacionales con Asia: La integración económica entre China y Colombia. Revista Internacional de Cooperación y Desarrollo, v. 4, n. 2, p. 106-121, 2017. Disponible en: https://revistas.usb.edu.co/index.php/Cooperacion/article/view/3340/2769. Consultado: 22 agosto 2020

SACHSE, Victor. Hegemonic Stability Theory: An Examination. 1989. Tesis (Doctorado en Ciencias Políticas), Louisiana State University and Agricultural \& Mechanical College, Louisiana, 1989. Disponible en: https://digitalcommons.Isu.edu/gradschool_disstheses/4740. Consultado: 20 agosto 2020

SAFE. China's External Portfolio Investment Assets by Country or Region. State Administration of Foreign Exchange, 2020. Disponible en: https://www.safe.gov.cn/en/ChinasExternalPortfoliolnvestm/index.html. Consultado: 8 agosto 2020. Consultado: 21 agosto 2020

SISTEMA ESTADÍSTICO DE COMERCIO EXTERIOR. Exportaciones e Importaciones por Clasificación Internacional Industrial Uniforme -CIIU-. 
Dirección de Impuestos y Aduanas Nacionales de Colombia (DIAN), 2020. Disponible en: http://websiex.dian.gov.co/. Consultado: 8 agosto 2020.

Taylor, Lance. (1990). Macro constraints on India's economic growth. En: Taylor, L. (Ed.), Socially Relevant Policy Analysis: Structuralist Computable General Equilibrium Models for the Developing World. MIT Press, 1990, cap. 8. p. 220-240.

UNCTAD. Gross Domestic Product: Total and Per Capita, Growth Rate, Annual. United Nations Conference on Trade and Development, 2020a. Disponible en: https://unctadstat.unctad.org/wds/TableViewer/tableView.aspx?Reportld=9 6. Consultado: 8 agosto 2020.

UNCTAD. Total Trade and Share, Annual. United Nations Conference on Trade and Development, 2020b. Disponible en: https://unctadstat.unctad.org/wds/TableViewer/tableView.aspx?Reportld=1 01. Consultado: 8 agosto 2020.

URIBE, José. D. El comercio exterior de Colombia con China. Revista del Banco de la República, v. 83, n. 995, p. 5-18, 2010. Disponible en: https://publicaciones.banrepcultural.org/index.php/banrep/article/view/847 0/8871. Consultado: 24 agosto 2020

VAN DE HAAR, Edwin. Classical Liberalism and International Relations Theory: Hume, Smith, Mises, and Hayek. USA: Palgrave Macmillan US, 2009. DOI: https://doi.org/10.1057/9780230623972. Consultado: 21 agosto 2020

WEBB, Michael. C.; KRASNER, Stephen. D. Hegemonic stability theory: an empirical assessment. Review of International Studies, v. 15, n. 2, p. 183-198, 1989. DOI: https://doi.org/10.1017/S0260210500112999. Consultado: 23 agosto 2020

WIENER, Jarrod. "Hegemonic" Leadership: Naked Emperor or the Worship of False Gods?. European Journal of International Relations, v. 1, n. 2, p. 219-243, 1995. DOI: https://doi.org/10.1177/1354066195001002004. Consultado: 25 agosto 2020

YARBROUGH, Beth; YARBROUGH, Robert. Free Trade, Hegemony, and the Theory of Agency. Kyklos, v. 38, n. 3, p. 348-364, 1985. DOl: https://doi.org/10.1111/j.1467-6435.1985.tb02231.x. Consultado: 28 agosto 2020

YAZID, Noor Mat. The Theory of Hegemonic Stability, Hegemonic Power and International Political Economic Stability. Global Journal of Political Science and Administration, v. 3, n. 6, p. 67-79, 2015. Disponible en: 
https://www.eajournals.org/journals/global-journal-of-political-science-andadministration-gjpsa/vol-3issue-6-december-2015/the-theory-of-hegemoni c-stability-hegemonic-power-and-international-political-economic-stabilit. Consultado: 18 agosto 2020. 